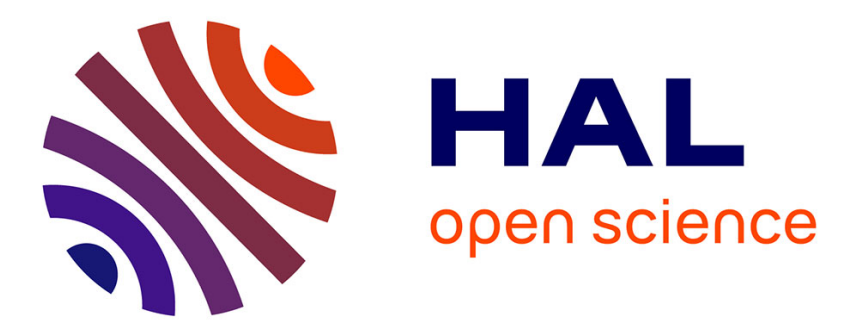

\title{
Ground Albedo Neutron Impacts to Seasonal Variations of Cosmic-Ray-Induced Neutron in Medium Geomagnetic Latitude and Antarctica: Impacts on Soft Error Rate
}

\author{
G. Hubert
}

\section{To cite this version:}

G. Hubert. Ground Albedo Neutron Impacts to Seasonal Variations of Cosmic-Ray-Induced Neutron in Medium Geomagnetic Latitude and Antarctica: Impacts on Soft Error Rate. IEEE Transactions on Nuclear Science, 2017, 64 (1), pp.622-629. 10.1109/TNS.2016.2614540 . hal-01487917

\section{HAL Id: hal-01487917 https://hal.science/hal-01487917}

Submitted on 13 Mar 2017

HAL is a multi-disciplinary open access archive for the deposit and dissemination of scientific research documents, whether they are published or not. The documents may come from teaching and research institutions in France or abroad, or from public or private research centers.
L'archive ouverte pluridisciplinaire $\mathbf{H A L}$, est destinée au dépôt et à la diffusion de documents scientifiques de niveau recherche, publiés ou non, émanant des établissements d'enseignement et de recherche français ou étrangers, des laboratoires publics ou privés. 


\title{
Ground Albedo Neutron impacts to Seasonal Variations of Cosmic-Ray-induced Neutron in medium Geomagnetic Latitude and Antarctica: Impacts on Soft Error Rate
}

\author{
G. Hubert
}

\begin{abstract}
This paper investigates the impact of ground albedo neutrons in the terrestrial radiation field and their seasonal variations. A modeling methodology is proposed, it is based on ATMORAD (atmospheric shower modeling) and GEANT4 simulations taking into account the soil characteristics and hydrogen content. This method was validated thanks to neutron measurements performed in two high altitude stations located in medium geomagnetic latitude and Antarctica (Pic-du-Midi and Concordia, respectively). Thus, the chemical rock composition, the density, the water content and the snow pack impacts to neutron spectrum were investigated. Simulated and measured spectra were compared. Analyses show that variations are dominant in the thermal domain (i.e. $\mathrm{En}<0.5 \mathrm{eV}$ ) and lesser degree in epithermal and evaporation domains (i.e. $0.5 \mathrm{eV}<\mathrm{En}<$ $0.1 \mathrm{MeV}$ and $0.1 \mathrm{MeV}<\mathrm{En}<20 \mathrm{MeV}$, respectively). The last part investigates the impact induced by the evaporation neutron contribution to Single Event Rate (more impacted by the ground albedo neutron). Results show that evaporation neutrons contribution to the total SER should be assessed for highsensitive technologies.
\end{abstract}

Index Terms - Cosmic-ray induced neutrons, ground albedo neutron, seasonal variations, GEANT4, high-altitude stations.

\section{INTRODUCTION}

In terrestrial fields, the Single Event Upset (SEU) occurence is attributed to thermal neutron, fast neutron and alpha emissions [1]-[4]. More recently, impacts of protons [5]-[9] and muons [10][11] were demonstrated. The evaluation of the failure rate requires one to associate the radiation field and the cross section deduced directly by experiments or simulations. As mentioned in the JEDEC JESD89A standard [12], for actual generations of devices, the neutron energy threshold has been characterized to be $10 \mathrm{MeV}$. Nevertheless, recent works describe the importance to take into account the low-energy threshold in determining an accurate estimate of Soft Error Rate (SER), particularly for nanoscale devices. Indeed, Yahagi et al. show in [13] that the energy neutron threshold can induce a significant difference in SER estimations for SRAM as small as the $130 \mathrm{~nm}$ technological node.

The precise knowledge of the fluence rate and spectrum of

Manuscript received July 11, 2016

This work is supported by the IPEV (French Polar Institute, Institut PaulEmile Victor) in the framework of the program no. 1112 (CHINSTRAP).

Guillaume Hubert is with the French Aerospace Laboratory (ONERA), Toulouse, France (phone: 33-562252885; fax: 33-562252569; e-mail: Guillaume.hubert@onera.fr). atmospheric cascade particles induced by galactic and solar cosmic rays is important. Secondary cosmic radiations are produced by the interaction of primary cosmic rays with the nuclei of the constituents of the atmosphere [7]. They can interact further with atoms and nuclei of the atmosphere to produce extensive showers of neutrons, protons, photons, electrons, positrons, pions, and muons. In the terrestrial environment, neutron fluence rates and spectra can be impacted by some mechanisms as the environmental water content (hydrometry, snow and vegetation) [14], the interaction of alpha particles emitted by radon [15] (underground environment), by the weather condition [16] or by seismic activities [17]. Besides, cosmic and terrestrial sources, atmospheric neutrons may be also be generated by lightning discharges [18].

Neutron detectors (commonly named neutron monitors NM, [19][20]) and neutron spectrometers are usually used to monitor the dynamics of incoming cosmic-ray neutrons of high energy (cascade neutron i.e. $\mathrm{E}>20 \mathrm{MeV}$ ) because local environmental conditions have no influences. However, neutrons below energy of $10-20 \mathrm{MeV}$ are most affected by the local environment. This fact is the result of the interaction of atmospheric neutrons with the ground matter, inducing secondary neutrons referred to as ground albedo neutrons. Hydrogen in soil, air and snow determines the amount of ground albedo neutron in the sensitive energy range from $1 \mathrm{eV}$ to $20 \mathrm{MeV}$. The investigation of ground albedo neutrons is not simple because neutron spectrum measurements are required over a long time allowing one to analyze the impact of seasonal changes.

A network composed by neutron spectrometers operated in high-altitude stations in medium geomagnetic latitude and Antarctica Environment has been active since 2011 [21]-[24]. More precisely, data recorded since 2011 were available in the Pic-du-Midi station [21], and since December 2015 in Concordia (Antarctica). To improve the data analyses, Hubert et al. presents in [22] an atmospheric radiation model named ATMORAD and based on simulations of Extensive Air Showers, the Force-Field Approximation to model the galactic cosmic rays (GCR) and the solar modulation potential. This approach is used to estimate the atmospheric radiation environment for terrestrial/atmospheric applications and to monitor the solar potential. Thus, the knowledge of variations of thermal (i.e. En $<0.5 \mathrm{eV})$, epithermal $(0.5 \mathrm{eV}<$ En $<0.1$ $\mathrm{MeV})$ and evaporation $(0.1 \mathrm{MeV}<\mathrm{En}<20 \mathrm{MeV})$ domains 
can improve cosmic radiation monitoring [25], and can be used to specify spectrum in SER calculations.

In this work, the main objective is to investigate the impact of ground albedo neutron in spectrum modifications, particularly in the thermal, epithermal and evaporation domains. In a first part, the impact of ground albedo neutrons was investigated using ATMORAD and GEANT4 simulations with the aim to improve the atmospheric model taking into account the impact of local environment. The approach was validated considering measured spectra from the Concordia and Pic-du-Midi stations. The second part is devoted to evaluate the impact of radiation field improvements on the SER calculations.

\section{EXPERIMENTS, METHODS AND SIMULATIONS}

\section{A. Neutron spectrometers network in High-Altitude stations}

In May 2011, a neutron spectrometer [24] extended to the high energy was firstly operated at the summit of the Pic-duMidi in the French Pyrenees (see table I and [22][23]). A second spectrometer was operated simultaneously since February 2015 at the summit of the Pico dos Dias (Observatory of Pico dos Dias) depending from the LNA (National Astrophysics Laboratory) in Brazil (table I). In the framework of the Continuous High-altitude Investigation of Neutron Spectra for Terrestrial Radiation Antarctic Project (CHINSTRAP) supported by IPEV (French Polar Institute), a third neutron spectrometer composed of six spheres was installed at Concordia research station (Antarctica) [21]. The station is located at Dome $\mathrm{C}$ on the Antarctic Plateau $\left(75^{\circ} 06^{\prime}\right.$ $\left.\mathrm{S}, 123^{\circ} 23^{\prime} \mathrm{E}\right)$ at height of 3,233 meters above the sea level. The site has an almost zero rigidity cutoff $(\mathrm{R}<0.01 \mathrm{GV})$, i.e., no geomagnetic shielding even for low-energy particles.

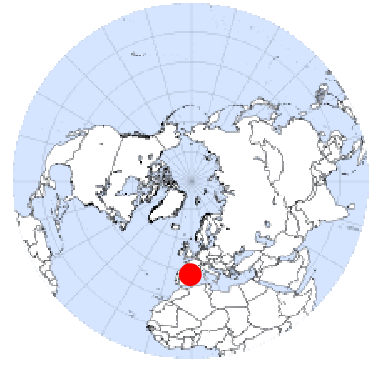

Pic-du-Midi [22][23][26]
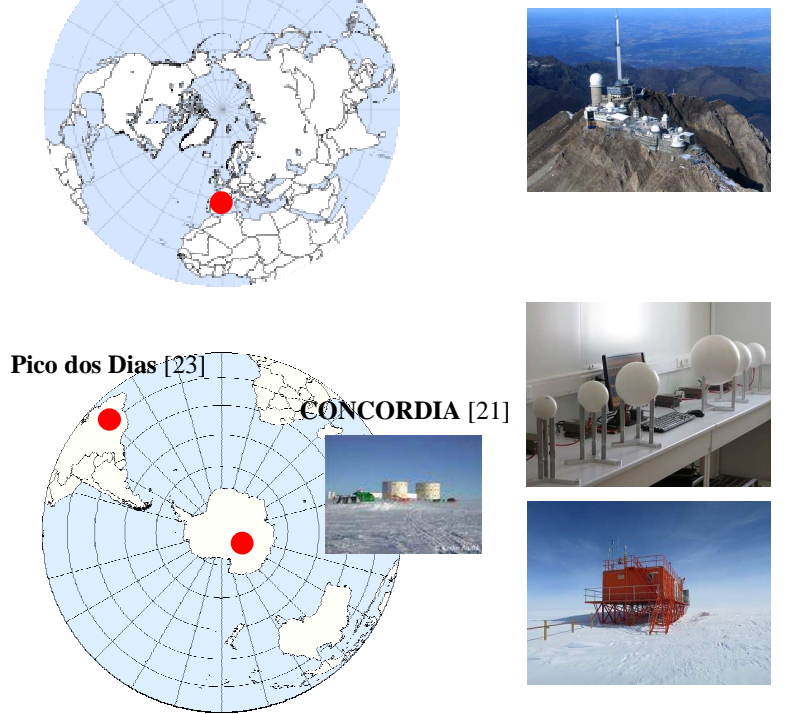

Fig. 1 : Location and view of the neutron spectrometer operated in Concordia since December 2015.
These three high-altitude stations constitutes a neutron spectrometer network which records simultaneously the neutron fields and their short/long time dynamics (i.e. seasonal variations and solar flare events). This work is exclusively based on data recorded in the Pic-du-Midi and Concordia stations. Measurements were used to validate the proposed modeling approach and to investigate the ground albedo neutron impact on radiation field variations. The ACROPOL platform has been presented in previous work [26], thus the Fig. 1 focus on the new installation in Concordia (location in Antarctica, the shelter, and the neutron spectrometer) while the table I summarizes station characteristics such as altitude, latitude and longitude.

TABLE I. CHARACTERISTICS OF PIC-DU-MIDI AND CONCORDIA STATIONS

\begin{tabular}{lll}
\hline & $\begin{array}{l}\text { Pic-du-Midi, } \\
\text { France }\end{array}$ & $\begin{array}{l}\text { Concordia station, } \\
\text { Antarctica }\end{array}$ \\
\hline Altitude & $2885 \mathrm{~m}$ & $3233 \mathrm{~m}$ \\
Latitude & $42^{\circ} 55^{\prime} \mathrm{N}$ & $75^{\circ} 06^{\prime} \mathrm{S}$ \\
Longitude & $0^{\circ} 08^{\prime} \mathrm{E}$ & $123^{\circ} 19^{\prime} \mathrm{E}$ \\
Cut-off rigidity & $5.6 \mathrm{GV}$ & $<0.001 \mathrm{GV}$ \\
\hline $\begin{array}{l}\text { Atmospheric } \\
\text { depth }\end{array}$ & $700.7 \mathrm{~g} / \mathrm{cm}^{2}$ & $635 \mathrm{~g} / \mathrm{cm}^{2}$ \\
\hline Start Operating & May 2011 & December 2015 \\
\hline
\end{tabular}

Data record by the neutron spectrometers consist in a series of counts $M_{d}$ recorded at the end of every cycles of tacq (five minutes). The fluence responses $\mathrm{R}_{\mathrm{d}}(\mathrm{E})$ (calculated by Monte Carlo simulations with the MCNPX2.6 and GEANT4 codes [24]) of the set of Bonner spheres are mandatory to derive the neutron differential fluence rate $\dot{\phi}_{E}(E)$ from a series of measurements $M_{d}$ during the acquisition time tacq (and an absolute uncertainty $\varepsilon_{d}$ ) with an unfolding procedure. Thus, equation 1 can be iteratively solved by using a code named GRAVEL starting with a default spectrum [27][28]

$$
\left(M_{\mathrm{d}} / t_{\mathrm{acq}}\right) \pm \varepsilon_{\mathrm{d}}=\int_{E_{\min }}^{E_{\max }} R_{\mathrm{d}}(E) \dot{\phi}_{E}(E) d E
$$

The relative uncertainty was investigated in [24] and it depends to number $\mathrm{N}_{\text {int }}$ of acquisition cycles and $\mathrm{M}_{\mathrm{d}}$, the objective consist in obtaining a compromise

\section{B. Atmospheric showers and ground albedo neutron modeling}

The using of physical Monte-Carlo simulations (GEANT4 in this work) allows one to investigate the neutron transport in the material environment. Thus, it is possible to integrate intrinsic characteristics (soil composition, porosity) and to take into account seasonal changes (snow, humidity in the rock). Usually the terrestrial radiation field is based on atmospheric showers physical modeling, and consideration of soil characteristics is basic or neglected.

Indeed, high-energy neutrons propagate through the atmosphere and generate low-energy neutrons by evaporation processes. The soil further slows neutrons down especially when water is present and they quickly moderate into the thermal regime. Fast neutrons which are reflected from the soil travel large distances before they get detected. Most of the 
detected neutrons originate from the first tens of meters around the sensor and are able to penetrate the soil down to $90 \mathrm{~cm}$. The total neutron spectrum at ground level will have two components: the spectrum of neutrons from atmospheric showers and the neutron spectrum albedo.

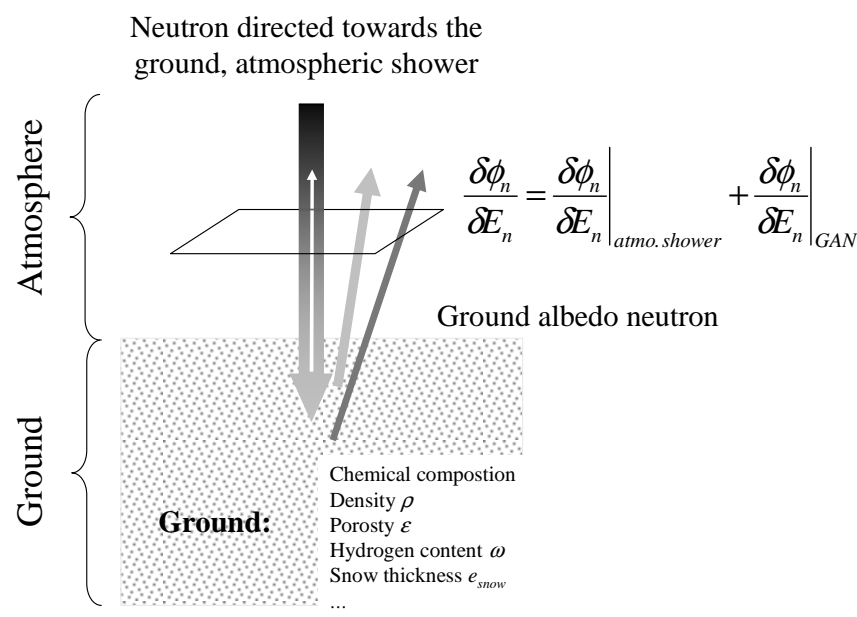

Fig. 2: Overview of the approach, the neutron spectrum result from the sum of atmospheric spectrum deduced from ATMORAD and ground albedo neutron calculated thanks to GEANT4 simulations.

As presented in Fig. 2, the general approach consists of considering a relatively simple scene geometry (ground plane), but to refine the level of detail in the modeling of soil with a description of the rock implying composition, density and porosity, humidity level then the incorporation of a layer of varying thickness snow. The neutron spectrum is the sum of the atmospheric spectrum deduced from ATMORAD and the ground albedo neutron spectrum calculated thanks to GEANT4 simulations

\section{1) Radiation field input data}

The first step before to quantify the impact of the local environment, is to define the incident neutron spectrum unmodified by the local environment (expect the atmosphere). The reference spectrum is provided by ATMORAD considering only downward-propagating neutrons. Thus, the backscattered particles are not considered and they will be replaced by the ground albedo neutron calculated from GEANT4 and considering the soil characteristics and dynamics (Pyrenees in the case of the Pic-du-Midi). Thus, a high number of particles (neutron and proton, according to the reference spectrum) are considered in GEANT4 simulations.

\section{2) Geometric modeling and physics}

The geometry of the simulated scene in GEANT4 consists of a series of rectangular shapes nested in one another, including the neutron/proton sources, the soil definition, the hydrogen content characteristics, the snow thickness and the atmosphere (defined as the air). As shown in Fig. 3, the volume "World" consists of vacuum is a cube whose edge is $200 \mathrm{~m}$. A smaller cube of $180 \times 180 \times 180 \mathrm{~m}^{3}$ is the volume of the atmosphere filled with air. The bottom half of this cube is occupied by a parallelepiped with a square face of $170 \times 170 \mathrm{~m}^{2}$ and $80 \mathrm{~m}$ deep. This volume represents the ground. This volume may reside another parallelepiped with same section but of variable thickness.

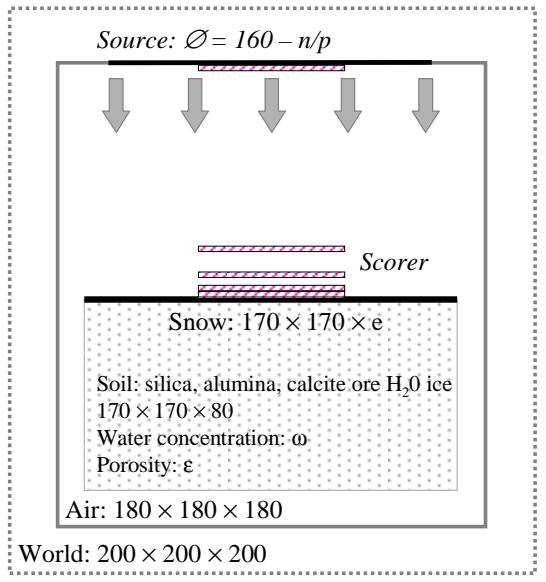

Fig. 3 : Description of the modeled scene from GEANT4. The elementary volumes representing the atmosphere, soil and snow are represented. The particle generation disc is also visible at the top of the atmosphere. The cylindrical volumes sensitive samplings at different altitudes are also presented (dimensions in meter).

Pic-du-Midi and Concordia characteristics are very different. The local environment in Concordia is well known and stable over time (i.e. only composed by ice and few precipitation levels). The Pic-du-Midi description is more complex because it is not simple to know precisely the geological compositions and hydrologic properties. Then, the strategy consists to determine the configuration "bare rock" (without snow) closest to the experimental results obtained in summer. Once the reference configuration defined, calculations by varying the snow thickness will allow deducing the spectra obtained during the winter.

The modeling is based on the GEANT4 code Version 10.1 developed by a World-wide collaboration and distributed by CERN [29]. The "emlowenergy" physical model is used; this physical model has been designed on top of Livermore physics for validation of new low-energy models. Intra-nuclear cascades are treated with the Bertini model (BERT), the cross sections of neutrons are high precision (HP) and finally the inclusion of thermal treatment of elastic scattering (TS: thermal scattering) in molecules of water is ensured.

\section{3) Modified spectrum calculation}

Neutron spectra for a given environment (i.e. $\omega, \rho$ and snow thickness) is equal to the contribution of the spectrum of the incident neutrons directed towards the ground and albedo backscattered or reissued by the ground neutrons. Four sensitive volumes were considerer, using volumetric fluence scorers (indicated $s$ ) located 1, 2, 5 and 100 meters above the scene, respectively. Each scorer allows one to calculate the spectral distribution $\phi_{k}^{g s}$ considering the atmospheric and the backscattered contributions, the index $g, k$ and $s$ denote the neutron energy group, the primary particle type (neutron or proton) and the selected sensitive volume. The last volume (i.e. $100 \mathrm{~m}$ ) is indented to discriminate the atmospheric shower contribution. 
The total fluence of incident particles $\phi_{\mathrm{n}}$ is derived from the number of particles and the area of the source disk using Equation 2, when $N_{k}$ is the total neutron or proton number considered in the simulation. The source was considered as isotropic over the upper hemisphere.

$$
\phi_{\mathrm{n}}=\mathrm{P}(k=n) \frac{N_{k}}{\pi \cdot R_{\text {source }}^{2}}
$$

Then, influence of the flux in each sensitive volume $\dot{\phi}_{n}^{s}$ is normalized to the conditions of the Pic-du-Midi or Concordia neutron spectrometer measured by equalizing the proportion of experimental cascade neutrons $\dot{\phi}_{\text {cas }}^{\text {Exp }}$ and determined from the unit spectrum given by ATMORAD $\dot{\phi}_{\text {cas }}^{A T M O R A D}$.

Through simulations, it is possible to calculate the modified neutron fluence rate considering the scorer $s$ and the neutron energy $E$ (equation 3 ). This is based on the local environment description with only a few input parameters i.e. the nature of the soil, the mass fraction of water in the rock, the porosity and the thickness of snow. $E^{g}$ and $\Delta E^{g}$ denote the neutron energy and the neutron energy range, respectively.

$$
\begin{aligned}
& \dot{\phi}_{n}^{s}\left(E^{g}, \omega, \rho, \varepsilon, e_{\text {snow }}, t\right)= \\
& \frac{\phi_{n}^{g s}\left(E^{g}, \omega, \rho, \mathcal{\varepsilon}, e_{\text {snow }}\right)}{\Delta E^{g}} \cdot \frac{\dot{\phi}_{\text {cas }}^{E x p}(t)}{\phi_{n} \cdot\left(\dot{\phi}_{\text {cas }}^{\text {ATMORAD }} / \dot{\phi}_{\text {tot }}^{\text {ATMORAD }}\right)}
\end{aligned}
$$

\section{GROUND ALBEDO NEUTRON IMPACT TO SPECTRUM VARIATIONS}

\section{A. Seasonal variation analyses}

Variations in the neutron daily fluence rate recorded in the Pic-du-Midi were presented in previous works [23] from June 2011 to April 2015. Updated measurements are presented in Fig. 4 until June 2016. Results show that total neutron flux variations are sinusoidal with a period of one year. This periodicity can be attributed to the local water/snow environmental dynamics. Snow cover and water content in the soil may alter the ground albedo neutrons and their transportation (absorption and backscattering). Then, it is possible to show [23] that seasonal oscillations are predominant in the thermal part and to a lesser extends on the epithermal and evaporation regions.

Indeed, the soil is wetter during the summer as a result of the higher precipitation level and melting winter snow. In winter, precipitation induces a snowfall of up to several meters thick. The soil is relatively dry because water does not infiltrate. Thus, at mountain altitude, the most abundant amount of hydrogen is provided by snowfall in winter.

The amount of snow deposited on the ground was measured in the Pyrenees at different altitudes by automatic stations Nivôse managed by Météo France. There are four stations in the mountains of the Pyrenees, with altitudes ranging from
$2140 \mathrm{~m}$ to $2445 \mathrm{~m}$. This is therefore a range of altitude close to the summit of the Pic du Midi although slightly lower (2885 $\mathrm{m}$ at the Pic-du-Midi). The data from these stations allowed estimation of the average variation over three decades (19812016) of accumulated snow depth.

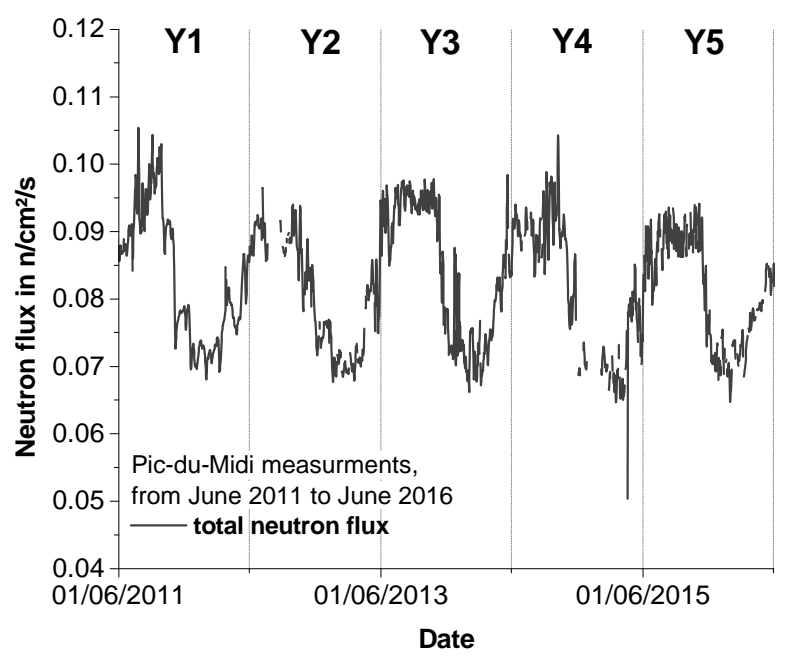

Fig. 4 : Neutron flux variations from June 2011 to June 2016 in the Pic-duMidi.

Thus, Fig. 5 presents the neutron flux recorded during the period 2011-2016 as function of the averaged thickness of accumulated snow. Neutron flux decreases exponentially with the snow thickness which is consistent with the neutron-matter interaction mechanisms. Moreover, it is interesting to note a low decrease in neutron flux from a snow thickness of approximately $100 \mathrm{~cm}$.

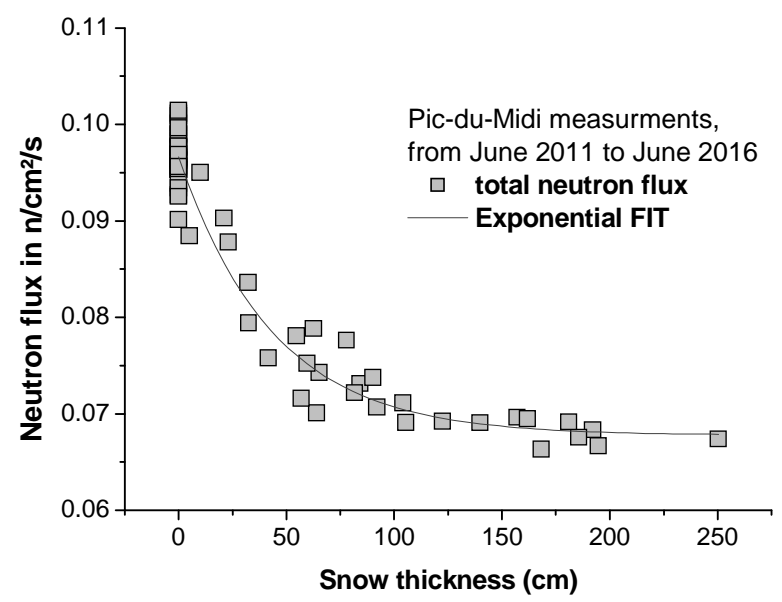

Fig. 5 : Neutron flux recorded from June 2011 to June 2016 in the Pic-duMidi, versus the averaged thickness of accumulated snow in the Pyrenees (measured closed to the Pic-du-Midi station).

\section{B. Ground albedo neutron modeling}

Impacts of the the chemical rock composition, its density and the water content were simulated to compare the calculated spectra and the measured spectra (summer). Then, a snowpack was added in the reference configuration to take 
into account the effect induced by the snow during the winter.

\section{1) Validation of the methodology based on Concordia data}

Concordia measurements are available since December 2015. The soil composition and the hydrogen content are well known and stable over time because there is little precipitation and the scene description can be reduced to an ice water thickness. The first measurements (December to June) can be used to assess the relevance of the modeling approach.

Thus, Table II presents the measured flux (average data from December to June 2016) and the calculated flux issued from GEANT4 simulations and for the four energy domains. In simulations, the "ground" volume was composed by $\mathrm{H}_{2} \mathrm{O}$ ice using $0.35 \mathrm{~g} / \mathrm{cm}^{3}$ density. The incident spectrum is issued from ATMORAD, considering the altitude, period, geomagnetic conditions and atmospheric description of Concordia. A good agreement were obtained between simulation and measured fluxes.

TABLE II. MEASURED AND CALCULATED FLUXES IN CONCORDIA

\begin{tabular}{ccc}
\hline Domain & $\begin{array}{c}\text { Measurements } \\
\text { from Dec. to June 2016 }\end{array}$ & $\begin{array}{c}\text { Simulation } \\
\mathrm{H}_{2} 0 \text { ice, density }=0.35 \mathrm{~g} / \mathrm{cm}^{3}\end{array}$ \\
\hline Thermal & $3.96 \mathrm{E}-02$ & $3.54 \mathrm{E}-02$ \\
Epithermal & $3.27 \mathrm{E}-02$ & $5.07 \mathrm{E}-02$ \\
Evaporation & $6.10 \mathrm{E}-02$ & $6.26 \mathrm{E}-02$ \\
Cascade & $5.19 \mathrm{E}-02$ & $5.61 \mathrm{E}-02$ \\
\hline
\end{tabular}

Analyses can be applied to the data obtained from the Picdu-Midi station, implying to define the rock composition, the porosity and the mass fraction of water and the snow thickness variations.

\section{2) Impact of the rock configuration}

To evaluate the effect of the chemical composition of the rock, four rocks referenced in table III were considered (without snow and moisture: $e_{\text {neigne }}=0$ and $\omega=0 \mathrm{~cm}$ ). The porosity $\varepsilon$ of the rock was set at 0.15 in the four cases.

TABLE III. MASS FRACTIONS OF SELECTED ROCKS

\begin{tabular}{cccc}
\hline Rock & $\mathbf{S i O}_{\mathbf{2}}$ & $\mathbf{A l}_{\mathbf{2}} \mathbf{O}_{\mathbf{3}}$ & $\mathbf{C a C O}_{\mathbf{3}}$ \\
\hline$\# 1$ & $75 \%$ & $25 \%$ & $0 \%$ \\
$\# 2$ & $70 \%$ & $14 \%$ & $17 \%$ \\
$\# 3$ & $49 \%$ & $8 \%$ & $43 \%$ \\
$\# 4$ & $46 \%$ & $0 \%$ & $54 \%$ \\
\hline
\end{tabular}

Thus, Fig. 6 shows fluxes obtained considering the four compositions and considering each energy domain. The influence of the nature of the modelled rock is relatively low in the output spectra (albedo + incident). This can be explained by the fact that the alumina and the calcite have similar molecular weights $(102 \mathrm{~g} / \mathrm{mol}$ and $100 \mathrm{~g} / \mathrm{mol}$, respectively). The main variation is observed for the epithermal neutron, but less than $5 \%$.

A first conclusion is that composition rock does not seem to be a major factor. However, the rock \#3 which is very close to the traditional granite, an inherent component to the Pyrenees, will be considered as a reference in the next analyses.

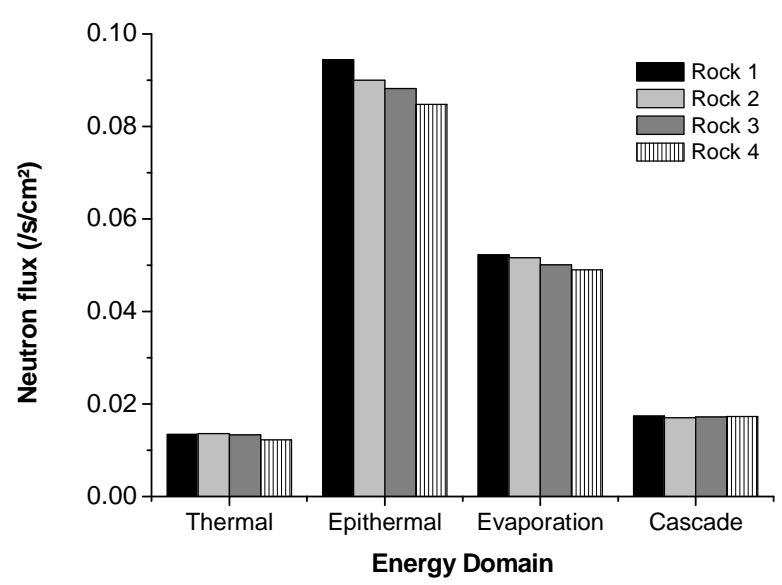

Fig. 6: Neutron flux in $/ \mathrm{s} / \mathrm{cm}^{2}$ for each energy domain, GEANT4 results obtained for 4 rocks chosen for their chemical composition.

3) Impacts of the porosity and the mass fraction of water

The impact of the porosity and the mass fraction of water were investigated. The porosity was considered equal to 0.3 , and the mass fractions of water $\omega$ were considered equal to 0 , $5,10,15$ and $20 \%$, respectively.

Neutron fluxes obtained for each energy domain (thermal, epithermal, evaporation and cascade) were presented in Fig. 7. A water content of $15 \%$ gives the closest spectrum of experimental configuration with evaporation peaks and cascading substantially identical, slightly higher epithermal region $(21 \%)$ and slightly lower thermal peak (-22\%). Fig. 8 shows neutron spectrum issued from measurements (summer) and optimal simulation (ATMORAD + GEANT4 ground albedo neutron) i.e. considering a porosity of 0.30 , and wet rocks of $15 \%$.

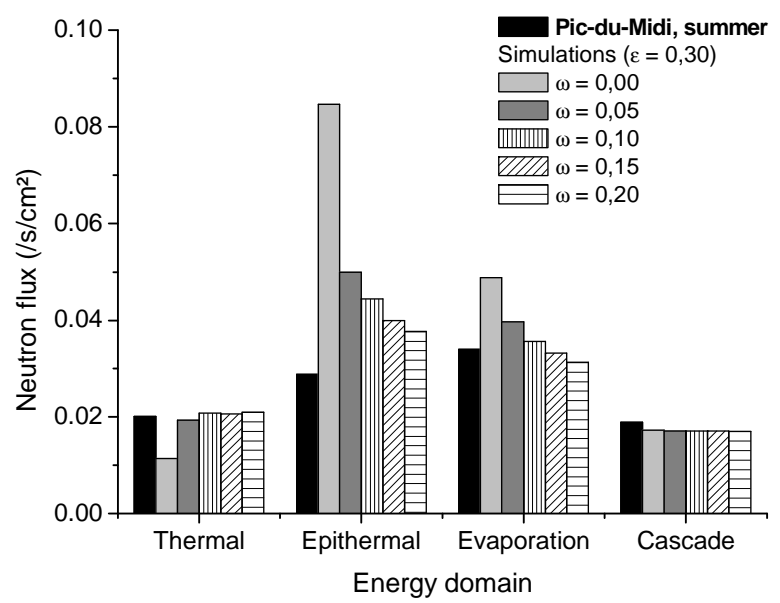

Fig. 7 : Neutron flux in $/ \mathrm{s} / \mathrm{cm}^{2}$ for each energy domain. Comparison between measurements and simulations considering a porosity of 0.30 and various wet rocks from 0 to $20 \%$. 


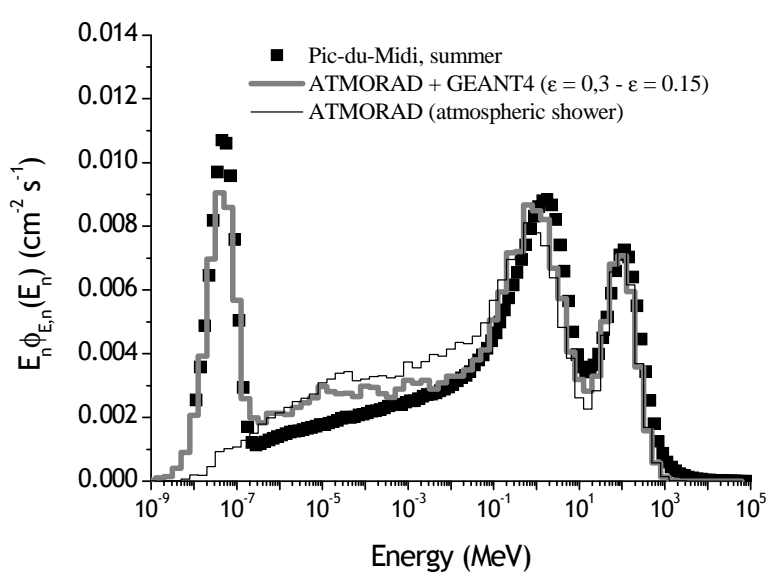

Fig. 8 : Neutron spectrum issued from measurements (summer) and simulation (ATMORAD + GEANT4 ground albedo neutron) considering a porosity of 0.30 and wet rocks of $15 \%$.

\section{4) Impacts of the snow thickness}

In this part, the impact of the addition of a snowpack (occurring during the winter) in the neutron spectrum is investigated.

Thus, a liquid water layer was considered in the simulation, the thickness of this layer varies from 5 to $300 \mathrm{~mm}$ to model the snow depth estimated at the Pic-du-Midi. An a priori equivalence of $1 \mathrm{~cm}$ of snow to $1 \mathrm{~mm}$ of rain was considered. The simulation results are shown in Fig. 9.

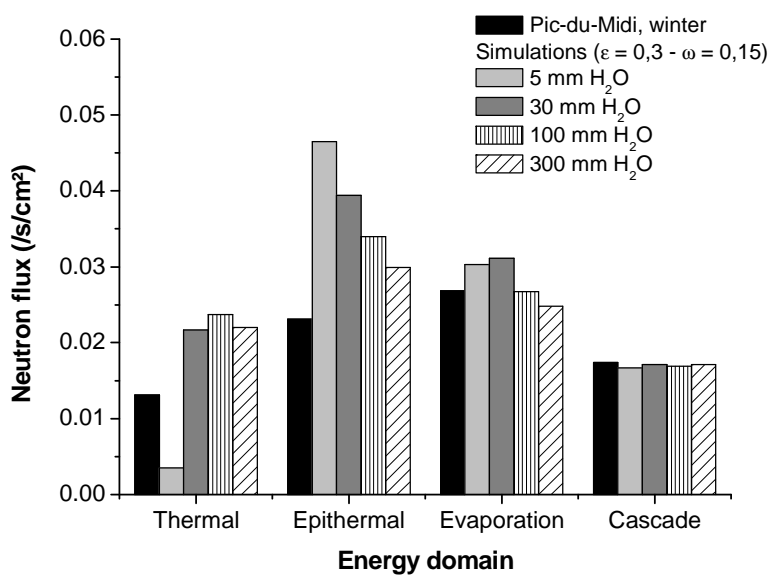

Fig. 9 : Neutron flux in $/ \mathrm{s} / \mathrm{cm}^{2}$ for each energy domain. Comparison between measurements and simulations considering a porosity of 0.30 , a wet rocks of $15 \%$ and for various liquid water layers with a thickness from 5 to $300 \mathrm{~mm}$. The experimental spectrum given by the spectrometer during wither is plotted.

Fluxes are compared with fluxes obtained experimentally with the neutron spectrometer in February 2015 (winter). The comparison is relevant for cascade and evaporation domains, while significant shifts were observed for thermal and epithermal domains. Thus, simulations overestimate the magnitude of the thermal peak (up to $55 \%$ for cases and 30 $\mathrm{mm}$ of water) and epithermal contribution.

Fig. 10 shows neutron spectrum issued from measurements (winter) and optimal simulation (ATMORAD + GEANT4 ground albedo neutron) i.e. considering a porosity of 0.30 , a wet rocks of $15 \%$ and a liquid water layer characterized by a thickness of $300 \mathrm{~mm}$.

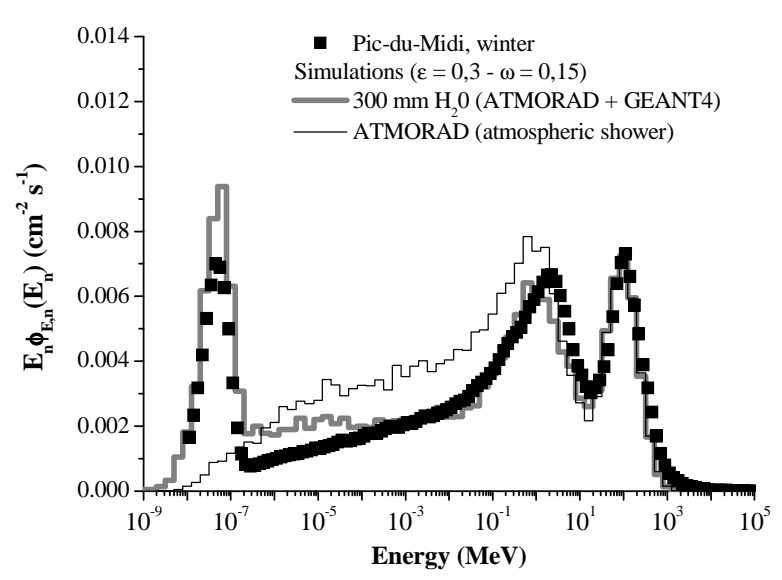

Fig. 10 : Neutron spectrum issued from measurements (winter) and simulation (ATMORAD + GEANT4 ground albedo neutron) considering a porosity of 0.30 a wet rocks of $15 \%$ and a liquid water layer with a thickness of $300 \mathrm{~mm}$.

\section{CONTRIBUtion OF THE GROUND ALBEDO NEUTRON MODELING TO THE SOFT ERROR RATE}

As feature sizes in nano-scale devices continue to shrink, the elementary charge (critical charge $\mathrm{Q}_{\text {crit }}$ ) required to induce an SEU continues to decrease. Indeed, the critical collected charge required to cause an error in a circuit reduces due to the reduction of operation voltage and capacitance of transistors. Previous works [31][32] provide critical charge estimation for current or future devices. Thus, nanoscale technologies are characterized by a critical charge which may be on the order of 0.1 fC. As shown in [33], neutron cross sections are significant for neutron energies below $10 \mathrm{MeV}$. This part of the paper is devoted to investigating the impact of the evaporation neutrons on SER rates, then to evaluating the improvement due to the presence of water.

Thus, a SRAM array composed by $100 \times 100$ cells in a 28 nm planar bulk CMOS has been considered using MUSCA SEP3 [34][35]. The modeling of radiation effects in nanoscale devices implied to take into account high level physical description. Thus, realistic secondary ion track 3D structures issued from GEANT4 simulations were considered [36]-[39]. Carriers and charges evolve according to mechanisms as well as drift (electric field), diffusion (carrier concentration gradient), collection and recombination processes.

Fig. 11 presents the FIT rate for the SRAM cells considering neutron spectra for the ground reference (NYC environment deduced from ATMORAD without soil description) and allowing to distinguish contributions induced by evaporation neutron $(<20 \mathrm{MeV})$ and by neutron energy less than $1 \mathrm{MeV}$. At low neutron energy, typically less than few $\mathrm{MeV}$, the elastic scattering is the predominant mechanism. For high-energy neutrons, SEUs are induced more by inelastic nuclear (spallation) collisions where the ionization arises from the combined effects of the recoiling in and 
secondary particles emitted from the nucleus.

The results clearly show that evaporation neutron contribute significantly to SER, around 10 to $25 \%$ considering the typical critical charge. Moreover, it is interesting to note that neutrons characterized by energies less than $1 \mathrm{MeV}$ induce a contribution of a few percent (not negligible but insufficient to present a significant additional problem). For the very low critical charge, this contribution reaches 5 to $10 \%$, but this is unrealistic from a technological point of view.

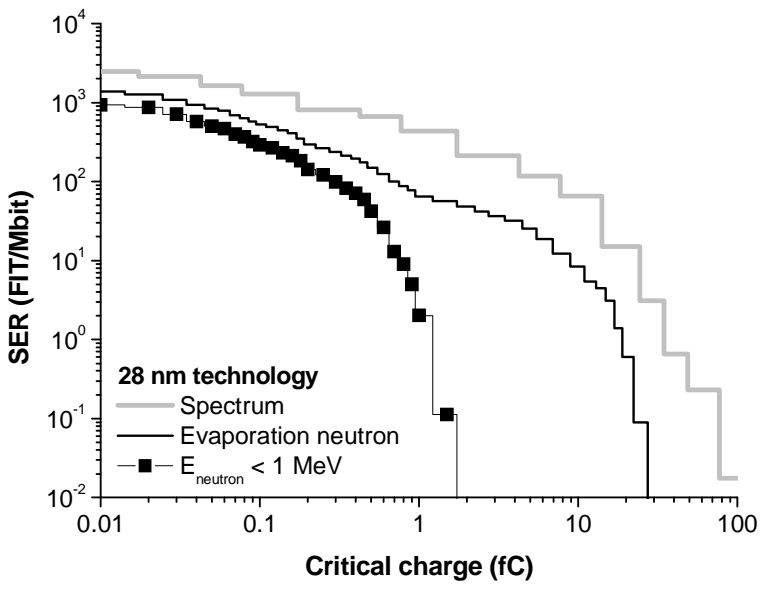

Fig. 11 : Predicted FIT rate for the $28 \mathrm{~nm}$ bulk CMOS cell considering neutron spectrum in ground and allowing to distinguish contributions induced by evaporation neutron $(<20 \mathrm{MeV})$ and by neutron energy less than $1 \mathrm{MeV}$.

The event multiplicity can be easily extracted from simulations. Multiple events represent about $20 \%$ of events when the spectrum was considered, while evaporation neutrons contribute to less than $2 \%$ of the SER. Thus, results presented in Fig. 11 indicate the significant impact of evaporation neutron; it is interesting to evaluate the interest to improve the neutron spectrum (i.e. the consideration of ground albedo neutron) in SER calculations.

Fig. 12 presents the predicted FIT rates obtained with a critical charge equal to $0.1 \mathrm{fC}$ and distinguishing the total, cascade and evaporation contributions. In accordance with Fig. 7, the soil porosity was considered equal to 0.3 and results were presented considering the mass fractions of water $\omega_{\max }$ from 0 to $20 \%$. Results show that the evaporation neutron contribution, although significant, induces relatively low impact on SER. This depends strongly on the critical charge, which in this case was considered realistic.

Fig. 13 presents the SER contribution induced by evaporation and cascade neutrons as function of the liquid water thickness from 0 to $300 \mathrm{~mm}$ and considering a critical charge equals to $0.1 \mathrm{fC}$. This result confirms the previous analyses, i.e. evaporation neutrons weakly affect the rate, on the order of 20 to $35 \%$. Whatever the hypothesis of hydrogen content in the soil (wet or liquid water thickness), the cascade neutrons are not impacted. This characteristic of quasi invariance with respect to the hydrogen environment was already used to monitor the cosmic ray from cascade neutron recording.

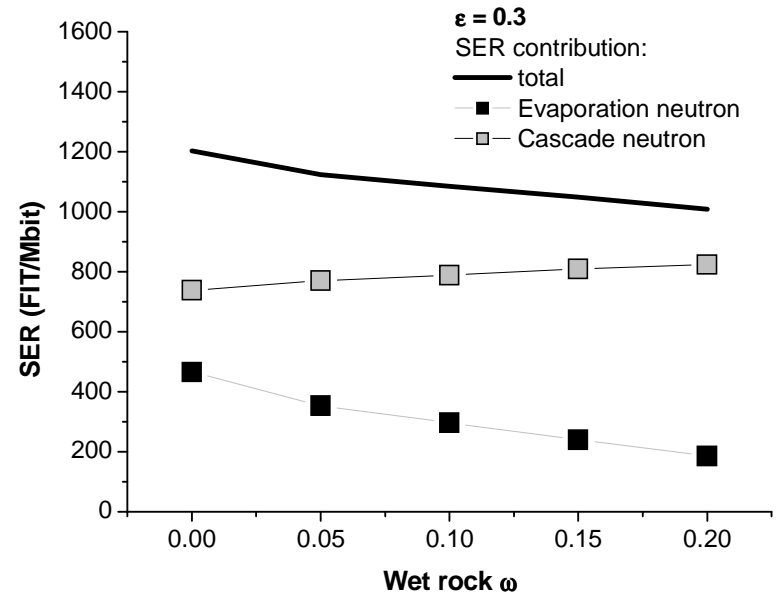

Fig. 12 : Predicted failure rate as function of the wet rock fraction, considering the neutron spectrum in ground (ATMORAD), and allowing to distinguish the evaporation contribution and the cascade contribution.

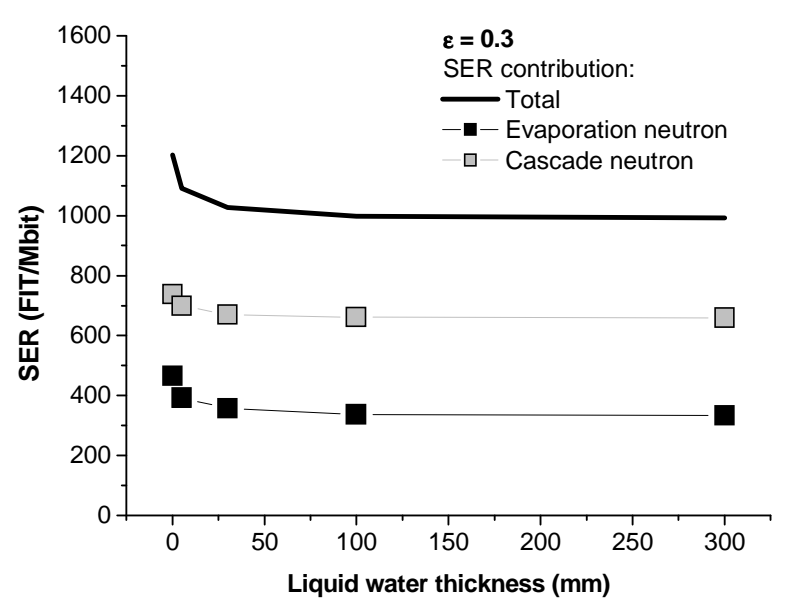

Fig. 13 : Predicted failure rate as function of the liquid water thickness, considering the neutron spectrum in ground (ATMORAD), and allowing to distinguish the evaporation contribution and the cascade contribution.

Similar analyses were conducted with low critical charges (i.e. $0.05 \mathrm{fC}$ ), which foreshadows future critical charge levels. Thus, the evaporation neutron contributions becomes more significant (up to $40 \%$ ), however the main contributor to SER are cascade neutrons. Then, evaporation neutrons constitute a large part of the terrestrial spectrum and their contribution to the total SER should be assessed for high-sensitive technologies. Moreover, to provide an exhaustive overview, it would be necessary to consider the alpha-SER, the proton and the muon contributions to SER.

\section{CONCLUSION}

This work investigates the ground albedo neutron influences on seasonal variations of the atmospheric neutron spectrum. The role of hydrogen content on ground albedo neutron generation was demonstrated with GEANT4 simulations considering soil description and hydrogen characteristics (porosity, wet, snow thickness). To validate the modeling 
approach and investigate the seasonal variations, this work is based on two neutron spectrometers operating in remote altitude stations, at the Pic-du-Midi and Concordia station, respectively.

Data analyses and GEANT4 simulations validate the role of hydrogen content in the ground albedo neutron generations. Thus, the modeled scene representative to the Pic-du-Midi was simulated for various hydrogen properties (composition, density, porosity and wet level) and snow thickness. The orders of magnitude of the calculated thermal fluence rate are consistent with measurements obtained during summers and winters. These investigations focused to the mountain environment and demonstrate the complex processes that need to be considered to quantify the ground neutron environment. A database which contains details of albedo neutrons (fluence, energy, and angle) was developed considering discrete neutron energies, the typical rock (granite, argils, snow/ice, etc.), the porosity and the humidity and the snow thickness. The coupling of ATMORAD and this database has improved the ground radiation field specifications for the terrestrial environment and the analyses of the ground neutron measurements.

The knowledge of variations of thermal, epithermal and evaporation domains can improve cosmic radiation monitoring. Indeed, variations implied corrections in the yield functions [25] of equipments (spectrometers or monitors). As shown in results, cascade neutrons $(\mathrm{En}>20 \mathrm{MeV})$ are weakly impacted, this property is usually used in cosmic ray analyses.

The last part investigates the contribution of the ground albedo neutron modeling to the SER. The results show clearly that evaporation neutron contribute significantly to SER, around 20 to $35 \%$ considering the typical critical charge. Moreover, neutrons characterized by energies less than $1 \mathrm{MeV}$ induce a contribution of a few percents (not negligible but insufficient to present a significant additional problem for SEU analysis). Similar analyses were conducted with low critical charges (i.e. $0.05 \mathrm{fC}$ ), which foreshadows future critical charge levels. Thus, the evaporation neutron contributions becomes more significant (up to $40 \%$ ), however the main contributor to SER are cascade neutrons.

\section{ACKNOWLEDGMENTS}

This work is supported by the IPEV (French Polar Institute, Institut Paul-Emile Victor) in the framework of the program n. 1112 (CHINSTRAP, Continuous High-altitude Investigation of Neutron Spectra for Terrestrial Radiation Antarctic Project).

The author thanks Dr. R. Cabanac, Mr. F. Lacassagne and the technical staff for their supports at the Pic-du-Midi station (OMP, Midi-Pyrenees Observatory). I'm thankful to the personnel of the Concordia station for their great help and hard work in harsh conditions, the scientific coordinator Doris Tuillier from IPEV and Luciano Milano.

\section{REFERENCES}

[1] P. Peronnard, R. Velazco and G. Hubert, "Real-life SEU experiments on 90nm SRAMs in Atmospheric Environment: measures vs. predictions done by means of MUSCA SEP3 platform", IEEE Trans. Nucl. Sci., Vol. 56, No. 6, pp. 3450-3455, Dec. 2009

[2] D. Lambert, J. Baggio, G. Hubert, V. Ferlet-Cavrois, O. Flament, "Neutron-induced SEU in SRAMs: Simulations with n-Si and n-O interactions", IEEE Trans. Nucl. Sci., Vol. 52, No. 6, pp. 2332-2339, Dec. 2005.

[3] D. Lambert, J. Baggio, G. Hubert, P. Paillet, S. Girard, "Analysis of Quasi-Monoenergetic Neutron and Proton SEU Cross Sections for Terrestrial Applications", IEEE Trans. Nucl. Sci., Vol. 53, No. 4, pp. 1890-1896, Aug. 2006.

[4] D. F. Heidel, K. P. Rodbell, E. H. Cannon, C. Cabral Jr., M. S. Gordon, P. Oldiges, H. H. K. Tang, "Alpha-particle-induced upsets in advanced CMOS circuits and technology", IBM J. RES. \& DEV., Vol. 52, No. 3, pp. 225-232, May 2008.

[5] K. P. Rodbell, D. F. Heidel, H. H. K. Tang, M. S. Gordon, P. Oldiges and C. E. Murray, "Low-energy Proton-Induced Single-event-Upsets in $65 \mathrm{~nm}$ node, Silicon-on-Insulator, Latches and Memory Cells", IEEE Trans. Nucl. Sci., vol. 54, no. 6, pp. 2474-2479, Dec. 2007.

[6] D. F. Heidel, P. W. Marshall, K. A. LaBel, J. R. Schwank, K. P. Rodbell, M. Hakey, M. D. Berg, P. E. Dodd, M. R. Friendlich, A. D. Phan, C. M. Seidleck, M. R. Shaneyfelt, and M. A. Xapsos, "Low energy proton single-event-upset test results on $65 \mathrm{~nm}$ SOI SRAM", IEEE Trans. Nucl. Sci., vol. 55, no. 6, pp. 3259-3264, Dec. 2008.

[7] B. D. Sierawski, J. A. Pellish, R. A. Reed, R. D. Schrimpf, K. M.Warren, R. A. Weller, M. H. Mendenhall, J. D. Black, A. D. Tipton, M. A. Xapsos, R. C. Baumann, X. Deng, M. J. Campola, M. R. Friendlich, H. S. Kim, A. M. Phan, and C. M. Seidleck, "Impact of lowenergy proton induced upsets on test methods and rate predictions", IEEE Trans. Nucl. Sci., vol. 56, no. 6, pp. 3085-3092, Dec. 2009.

[8] G. Hubert, S. Duzellier, F. Bezerra and R. Ecoffet, "MUSCA SEP3 contributions to investigate the direct ionization proton upset in $65 \mathrm{~nm}$ technology for space and atmospheric applications", Radiation and Its Effects on Components and Systems, Proc. RADECS 2009, Bruges, Belgium, Sept. 14-18, pp. 179-186.

[9] G. Hubert, S. Bourdarie, S. Duzellier, R. Ecoffet, "Impact of the Solar Flares on the SER dynamics on Micro and Nanometric Technologies in the Geostationary Orbit", IEEE Trans. Nucl. Sci., Vol. 57, No. 6, pp. $3127-3134$, Dec. 2010.

[10] B. D. Sierawski M. H. Mendenhall, R. A. Reed, M. A. Clemens, R. A. Weller, R. D. Schrimpf, E. W. Blackmore, M. Trinczek, B. Hitti, J. A. Pellish, R. C. Baumann, S. J. Wen, R. Wong, and N. Tam, "MuonInduced Single Event Upsets in Deep-Submicron Technology", IEEE Trans. Nucl. Sci., Vol. 57, No. 6, pp. 3273-3278, Dec. 2010.

[11] G. Hubert, L. Artola and D. Regis, "Impact of scaling on the soft error sensitivity of bulk, FDSOI and FinFET technologies due to atmospheric radiation", Integration, the VLSI journal, Vol. 50, pp. 39-47, June 2015.

[12] JESD89A: Measurement and reporting of alpha particle and terrestrial cosmic ray-induced soft errors in semiconductor devices. JEDEC Solid State Technology Association, 2006.

[13] Y. Yahagi, E. Ibe, Y. Takahashi, Y. Saito, A. Eto, M. Sato, H. Kameyama, M. Hidaka, K. Terunuma, T. Nunomiya, and T. Nakamura, "Threshold energy of neutron-induced single event upset as a critical factor", 42th Annual International Reliability Physics Symposium, Phoenix, pp. 669-670, April 2004.

[14] M. Schron, S. Zacharias, M. Kohli, J. Weimar, P. Dietrich, "Monitoring Environmental water with ground albedo neutrons and correction for incoming cosmic rays with neutron monitor data", 34th International Cosmic Ray Conference, The Hague, Netherlands, 30 July - 4 August 2015, ICRC2015-I/970.

[15] P. K. F. Grieder, "Cosmic Rays at Earth: Researcher's Reference Manual and Data Book" $1^{\text {st }}$ ed., pp. 1093, Elsevier, 2001.

[16] B. M. Kuzhevskij, O. Y. Nechaev, M. I. Panasyu, E. A. Sigaeva, N. N. Volodichev and V. A. Zakharov, "Neutron field of the Earth: Origin and dynamics", J. Korean Assoc. Radiat. Prot., Vol. 26, No. 3, pp. 315-319, 2001.

[17] J. A. Lockwood and H. E. Yingst, "Correlation of meteorological parameters with cosmic ray intensities", Phys. Rev., Vol. 104, Issue 6, 1718-1722, Dec. 1956.

[18] N. N. Volodichev, V. A. Zakharov, B. M. Kuzhevskij, O. Y. Nechaev, A. N. Podorolski, A. P. Chubenko, A. L. Sh'epetov, and V. P. Antonova, "The flows of neutrons of space radiation and from terrestrial crust", Proc. 27th International Cosmic Ray Conference, Int. Union of Pure and Appl. Phys., Hamburg, Germany, 2001, vol. 10, pp. 4204-4207. 
[19] S. Oh, J. W. Bieber, P. Everson, J. Clem, Y. Yi and Y. Kim, "Record Neutron Monitor Counting Rates from Galactic Cosmic Rays", J. Geophys. Res., Vol. 118, pp. 5431-5436, 2013.

[20] F. B. McDonald, "Integration of neutron monitors data with spacecraft observation: a historical perspective", Space Science Reviews, Vol. 93, Issue 1, pp. 239-258, June 2000.

[21] G. Hubert, "Analyses of Cosmic Ray induced-Neutron based on spectrometers operated simultaneously at mid-latitude and Antarctica high-altitude stations during quiet solar activity", Astroparticle Physics, Vol. 83, pp. 30-39, Oct. 2016.

[22] G. Hubert and A. Cheminet, "Radiation effects investigations based on atmospheric radiation model (ATMORAD) considering GEANT4 simulations of extensive air showers and solar modulation potential", Radiation Research, Vol. 184, No. 1, pp. 83-94, July 2015.

[23] G. Hubert, C. A. Federico, M. T. Pazianotto and O. L. Gonzales, "Long and short-term Atmospheric Radiation analyses based on coupled Measurements at High Altitude remote Stations and extensive Air Shower modeling", Astroparticle Physics, Vol. 74, pp. 27-36, Feb. 2016.

[24] A. Cheminet, V. Lacoste, V. Gressier, G. Hubert, A. Martin and M. Pépino, "Characterization of the IRSN neutron multisphere spectrometer (HERMEIS) at European standard calibration fields", IOP Science Journal of Instrumentation, 2nd International workshop on Fast Neutron Detectors and Applications (FNDA), Vol. 7, 2012, article C04007.

[25] J. Clem, "Atmospheric yield functions and response to secondary particles of neutron monitors", proceedings of 26th International cosmic Ray Conference (ICRC), pp. 317-320, 1999.

[26] G. Hubert, R. Velazco, C. Frederico, A. Cheminet, C. Silva-Cardenas, L. V. E. Caldas, F. Pancher, V. Lacoste, F. Palumbo, W. Mansour, L. Artola, F. Pineda and S. Duzellier, "Continuous high-altitude measurements of cosmic ray neutron and SEU/MCU at various locations: correlation and analyses based-on MUSCA-SEP3", IEEE Trans. Nucl. Sci., Vol. 60, No. 4, pp. 2418-2426, Aug. 2013.

[27] M. Matzke, "Unfolding procedures", Radiation Protection Dosimetry, Vol. 107, No. 1-3, pp. 155-174, 2003.

[28] M. Matzke, "Unfolding of Pulse Height Spectra: The HEPRO Program System", Report PTB-N-19, Physicalish-Technische-Bundensantalt, Braunshweig, 1994

[29] GEANT4 collaboration. Physics Lists EM constructors in Geant4 10.1, 2015 .
[30] L. M. Libby and H. R. Lukens, "Production od radiocarbon in tree rings by lightning bolts", J. Geophys. Res., Vol. 78, No. 26, pp. 5902-5903, Oct. 1973.

[31] B. D. Sierawski, R. A. Reed, M. H. Mendenhall, R. A. Weller, R. D. Schrimpf, S. J. Wen, R. Wong, N. Tam, R. C. Baumann, "Effect of scaling on Muon-Induced Soft Errors", in Proc. IEEE Int. Rel. Phys. Symp., pp. 247-252, Apr. 2011.

[32] E. Ibe, H. Taniguchi, Y. Yahagi, K.Shimbo, T. Toba, "Impact of scaling on neutron-induced soft error in SRAMs from a $250 \mathrm{~nm}$ to a $22 \mathrm{~nm}$ design rule", IEEE Tans. Elec. Dev., Vol. 57, No. 7, pp. 1527-1538, July 2010.

[33] Y. Yahagi, E. Ibe, Y. Takahashi, Y. Saito, A. Eto, M. Sato, H. Kameyama, M. Hidaka, K. Terunuma, T. Nunomiya, and T. Nakamura, "Threshold energy of neutron-induced single event upset as a critical factor", in Proc. IEEE Int. Rel. Phys. Symp., pp. 669-670, April 2004.

[34] G. Hubert, S. Duzellier, C. Inguimbert, C. Boatella-Polo, F. Bezerra, and R. Ecoffet, "Operational SER calculations on the SAC-C orbit using the Multi SCAles Single Event Phenomena Predictive Platform (MUSCA SEP3)", IEEE Trans. Nucl. Sci., Vol. 56, No.6, pp. 3032-3042, Dec. 2009.

[35] G. Hubert and L. Artola, "Single-Event Transient Modeling in a 65-nm Bulk CMOS Technology based-on Multi-Physical Approach and Electrical Simulations", IEEE Trans. Nucl. Sci., Vol. 60, No. 6, pp. 4421-4429, Dec. 2013

[36] M. Raine, G. Hubert, M. Gaillardain, L. Artola, P. Paillet, S. Girard, P. E. Sauvestre, A. Bournel, "Impact of the Radial Ionization Profile on SEE Prediction for SOI Transistors and SRAMs Beyond the 32-nm Technological Node", IEEE Trans. Nucl. Sci., Vol. 58, No. 3, pp. 840847, June 2011.

[37] M. Raine, G. Hubert, M. Gaillardin, P. Paillet, A. Bournel, "Monte Carlo Prediction of Heavy Ion Induced MBU Sensitivity for SOI SRAMs Using Radial Ionization Profile", IEEE Trans. Nucl. Sci., Vol. 58, No. 6, pp. 3127 - 3134, Dec. 2011.

[38] M. Raine, G. Hubert, P. Paillet, M. Gaillardin, A Bournel, "Implementing Realistic Heavy Ion Tracks in a SEE Prediction Tool: Comparison Between Different Approaches", Nuclear Science, IEEE Trans. Nucl. Sci., Vol. 58, No. 4, pp. 950 - 957, August 2012.

[39] G. Hubert, P. Li Cavoli, C. Federico, L. Artola and J. Busto, "Impact of the Radial Ionization Profile of proton on SEU Sensitivity of nanoscale SRAMs", IEEE Trans. Nucl. Sci., Vol. 62, No. 6, pp. 840-847, Dec. 2015 . 